\title{
Mesures LIF résolues dans le temps de la température et de l'épaisseur d'un film liquide à instabilités de surface ruisselant sur une paroi inclinée chauffée
}

\section{LIF measurements resolved in time of temperature and thickness of a liquid film with surface instability flowing down an inclined surface}

\author{
Romain COLLIGNON ${ }^{1 *}$, Ophélie CABALLINA ${ }^{1}$, Guillaume CASTANET ${ }^{1}$, Fabrice LEMOINE ${ }^{1}$ \\ ${ }^{1}$ LEMTA, Université de Lorraine, CNRS, Vandoeuvre-les-Nancy, France \\ * (auteur correspondant : romain.collignon@univ-lorraine.fr)
}

RÉSUMÉ. Une technique de mesure optique a été développée afin de caractériser les transferts de chaleur dans un film ruisselant mince tombant sur un plan incliné. Le film est perturbé par des vagues excitées harmoniquement et se propageant à sa surface libre. Une technique de Fluorescence Induite par Laser est spécialement développée afin de pouvoir mesurer de façon simultanée l'épaisseur et la température dans les vagues au cours du temps. Les mesures révèlent ainsi l'évolution de la température moyenne dans l'épaisseur du film au passage des vagues dont la fréquence peut être contrôlée.

ABSTRACT. An optical measurement technique has been developped to characterize the heat transfer in a falling liquid film with surface instabilities, which is flowing down an inclined plane. The waves traveling at the free surface are generated harmonically. The novel technique based on Laser Induced Fluorescence enables to provide simultaneously a measurement of the thickness and the temperature of the liquid film. The experimental results reveal the temporal evolution of the average temperature in the thickness of the film, when the film is travelled by waves of controlled frequency.

MOTS-CLÉS. Transferts de chaleur, Fluorescence induite par laser, Film ruisselant mince, Instabilités de surface.

KEYWORDS. Heat Transfer, Laser Induced Fluorescence, Thin falling film, Wave instabilities.

\section{Nomenclature}

a diffusivité thermique, $\mathrm{m}^{2} / \mathrm{s} \quad \Gamma$

C concentration, $\mathrm{mol} / \mathrm{L}$

$f \quad$ fréquence d'excitation, $\mathrm{Hz}$

$g$

I

$k$

$\mathrm{Nu} \quad$ nombre de Nusselt

$q_{w}$

$\dot{Q}$

$R$

$R e$

$s$

$T \quad$ température, $\mathrm{K}$

$\vec{U} \quad$ vitesse, $\mathrm{m} / \mathrm{s}$

$V \quad$ volume, $\mathrm{m}^{3}$

Symboles grecs

$\beta \quad$ angle d'inclinaison
$\Gamma \quad$ débit volumique par unité de largeur, $\mathrm{m}^{2} / \mathrm{s}$

$\delta \quad$ épaisseur du film, $m$

$\epsilon \quad$ coefficient d'absorption, $\mathrm{L} / \mathrm{m} / \mathrm{mol}$

$\sum \quad$ Somme d'intensités de fluorescence,

$\theta \quad$ Température réduite adimensionnée

$\lambda$ longueur d'onde, $\mathrm{nm}$

viscosité cinématique, $\mathrm{m}^{2} / \mathrm{s}$

$\phi$ rendement quantique de fluorescence

Indices et exposants

c contrôle

fl débitant

$i \quad$ colorant

las longueur d'onde du laser

ref référence

vol volumique

$w \quad$ paroi 


\section{Introduction}

Les films minces liquides sont utilisés dans de nombreux procédés mettant en jeu des transferts de chaleur et de masse, comme des condenseurs, des évaporateurs ou des colonnes d'absorption. En général, les conditions rencontrées dans ces applications sont telles que la surface libre du film est instable et des vagues s'y propagent. Ces vagues sont depuis longtemps utilisées pour intensifier les transferts de chaleur et de masse à travers le film [FRI 72]. Cependant, les mécanismes à l'origine de cette intensification ne sont pas encore complètement identifiés, le fort couplage entre l'hydrodynamique et les transferts de chaleur rendant la modélisation particulièrement complexe. L'augmentation de la surface d'échange entre le liquide et le gaz du fait des vagues, la formation de rouleaux dans le front de vague et l'amincissement du film devant la vague sont parmi les explications qui ont été le plus souvent avancées pour expliquer l'intensification des transferts [POR 71][RUY 14], mais toutes ces hypothèses restent encore à valider. Expérimentalement, la caractérisation des transferts dans ce type d'écoulement est une gageure due à l'épaisseur sub-millimétrique du film. Les techniques de mesure traditionnelles comme les sondes thermocouples ne sont pas appropriées. L'utilisation de techniques de mesure optique est indispensable en raison de leur caractère faiblement intrusif, mais la présence d'une interface déformée et mobile à la surface libre peut provoquer de fortes distorsions optiques. De fait, les diagnostics optiques employés ne permettent généralement pas d'accéder aux informations à l'intérieur du film mais uniquement en surface [ALS 02][MAR 16][MAT 13]. Il s'agit le plus souvent de l'utilisation de la thermographie infrarouge dans une bande opaque de l'eau. A notre connaissance, seul un petit nombre d'étude ont entrepris de mesurer la température ou la concentration à l'intérieur même de ces films sub-millimétriques [SCH 06][SCH 07], ce qui limite notre compréhension des mécanismes de transferts mis en jeu.

Dans cette étude, les transferts de chaleur sont étudiés dans un film liquide s'écoulant sur un plan incliné chauffé et soumis à une excitation harmonique. La température et l'épaisseur sont caractérisées par une technique basée sur la fluorescence induite par laser (LIF). La mesure de ces grandeurs est résolue dans le temps en un point et elle est en pratique faiblement perturbée par la déformation de l'interface gaz-liquide. La longueur d'onde des vagues résultantes de l'excitation harmonique étant très grande, de l'ordre de la dizaine de centimètre, l'angle d'inclinaison ainsi que la courbure de l'interface sont suffisamment faibles pour ne pas perturber la mesure. Dans ces expériences, un certain nombre de paramètres comme l'angle d'inclinaison du plan, le nombre de Reynolds de l'écoulement, la fréquence et l'amplitude des vagues peuvent être contrôlés pour étudier l'effet de ces paramètres sur le transfert de chaleur.

\section{Montage expérimental}

\subsection{Génération du film mince}

Le montage utilisé pour étudier le film ruisselant chauffé est illustré en figure 1. Le film ruisselant s'écoule sur un plan constitué d'une feuille de titane de $100 \mu \mathrm{m}$ d'épaisseur et de dimensions $30 \mathrm{~cm}$ de large et $40 \mathrm{~cm}$ de long. Le circuit hydraulique fonctionne en boucle fermée grâce à une pompe faisant circuler le liquide entre un réservoir tampon et un réservoir amont. Le débit qui est régulé par un by-pass, est mesuré par un débitmètre à ultrasons. Le film liquide est généré par le débordement du réservoir 
amont et dévale naturellement la pente du plan incliné. L'inclinaison de l'ensemble réservoir amont plan est ajustable à l'aide d'un pivot comme montré en figure 1. Le forçage des vagues se fait à l'aide de deux haut-parleurs. Une plaque de PVC, collée sur les membranes des deux haut-parleurs, est mise en contact avec la surface libre du liquide dans le réservoir supérieur, ce qui permet de communiquer son mouvement sinusoïdal de translation au liquide et ainsi de créer des vagues régulières. La fréquence de l'oscillation est contrôlée par la tension d'alimentation des haut-parleurs. L'amplitude des vagues est une conséquence du débit, de l'inclinaison et de la fréquence d'excitation.

Le plan incliné est chauffé par effet Joule. La feuille de titane est maintenue entre deux électrodes de cuivre, maintenues écartées pour tendre la feuille de métal et s'assurer de sa planéité ainsi que de sa rigidité. Les électrodes sont connectées à plusieurs sources de tension ajustables. La densité de courant traversant la feuille est quasiment uniforme, ce qui permet de considérer une densité de flux de chaleur homogène au niveau de la surface de travail du plan. Dans ces expériences la densité de flux surfacique de chaleur $q_{w}$ est fixée à $1,25 \mathrm{~W} / \mathrm{cm}^{2}$. Afin de contrôler la température d'injection du film, le réservoir tampon qui récupère le liquide ayant parcouru le plan, est équipé d'un échangeur de chaleur. Ce dernier refroidit le liquide échauffé pour arriver à maintenir une température d'injection constante au cours des mesures en régime établi. De très petits thermocouples de diamètre $0,2 \mathrm{~mm}$ sont fixés à l'arrière de la paroi pour mesurer la température de la paroi $T_{w}$.

Les équipements de ce montage permettent une variation de l'angle d'inclinaison $\beta$ entre 0 et $10^{\circ}$, du débit volumique $\dot{Q}$ entre 0 et 10 1/min et de la fréquence d'excitation $f$ entre 0 et $10 \mathrm{~Hz}$. Le liquide utilisé est de l'eau pure ensemencée par des colorants fluorescents à très faible concentration (typiquement quelques $\mu \mathrm{g} / \mathrm{L})$.

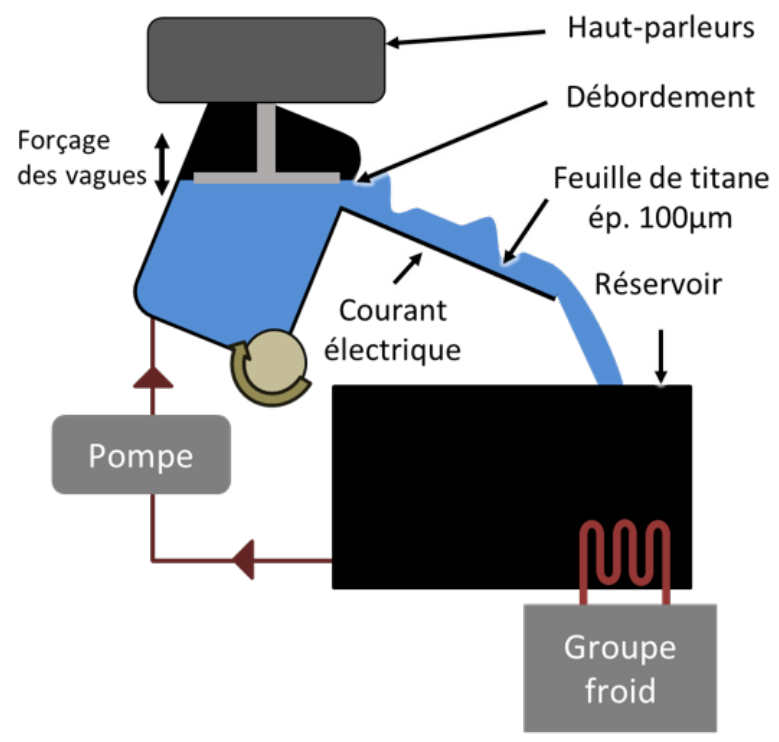

Figure 1. Montage expérimental d'étude des films ruisselants perturbés harmoniquement

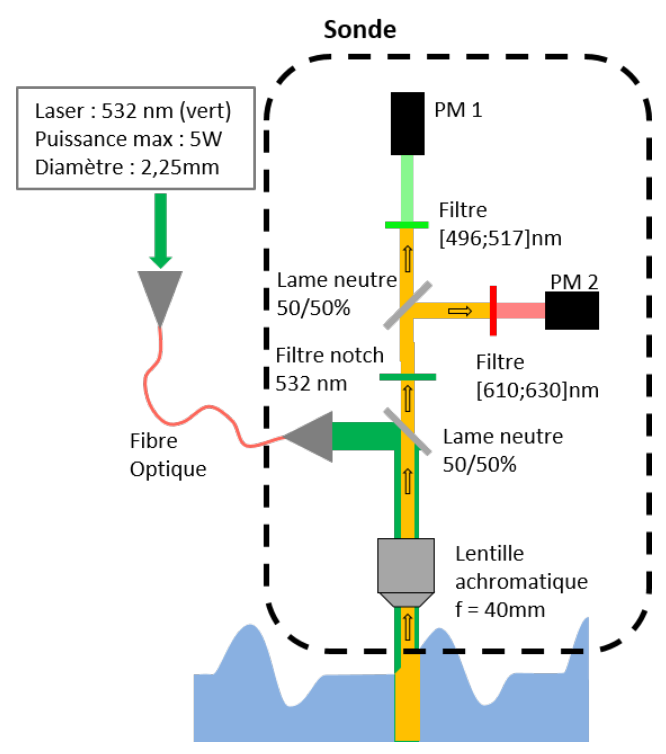

Figure 2. Montage optique pour la technique 2cLIF

\subsection{Mesure de température et d'épaisseur par LIF}

Afin de mesurer la température du liquide, une technique de mesure basée sur la fluorescence induite par laser (LIF) a été développée comme présenté en figure 2. La mesure se présente comme une 
adaptation de la technique LIF à deux couleurs et deux colorants déjà utilisée pour caractériser le champ de température dans des jets liquides turbulents, des impacts de gouttes sur des parois chaudes. Une description de la méthode et de son développement peut être trouvée dans des articles récents [STI 19][CHA 17][CHA 16][LEM 13]. La technique peut être déclinée sous plusieurs formes. Des mesures résolues dans le temps en un point où est focalisé un laser continu, peuvent être obtenues grâce à l'utilisation de photo-mutliplicateurs pouvant détecter le signal avec un taux d'échantillonage très élevé (plusieurs $\mathrm{MHz}$ ). Des mesures d'imagerie sont également possibles en utilisant des caméras et un laser pulsé. Cette étude se limite à des mesures ponctuelles de température dans l'épaisseur du film, ce qui constitue déjà un développement significatif.

Le liquide, ici de l'eau, est ensemencé par un couple de colorants fluorescents spécifiques (Rhodamine 560 et Kiton red). L'absorption d'une radiation laser par une molécule d'un colorant fluorescent induit une transition d'un état électronique fondamental vers un état électronique excité. L'absorption est suivi d'une désexcitation radiative, qui correspond à l'émission de fluorescence. Pour un certain nombre de colorants organiques, le spectre d'émission de la fluorescence est dépendant de la température. Cette dépendance affecte à la fois l'intensité globale du signal de fluorescence, mais aussi la réponse spectrale (forme du spectre de fluorescence). En négligeant l'atténuation du laser et de la fluorescence sur les chemins optiques, l'intensité du signal de fluorescence $I_{i, \lambda}$ émis dans un volume $V_{c}$ par un colorant $i$ à la longueur d'onde $\lambda$ peut être exprimée par :

$$
I_{i, \lambda}(T) \propto C_{i} V_{c} I_{l a s} \epsilon_{i, l a s}(T) \phi_{i, \lambda}(T)
$$

avec $C_{i}$ la concentration molaire du colorant $i, V_{c}$ le volume de collection, $I_{\text {las }}$ l'intensité du laser dans $V_{c}$, $\epsilon_{i, l a s}$ le coefficient d'absorption molaire du colorant $i$ à la longueur d'onde du laser et $\phi_{i, \lambda}$ le rendement quantique de fluorescence du colorant $i$ à la longueur d'onde $\lambda$. La sensibilité à la température du signal de fluorescence est essentiellement le résultat des désactivations collisionnelles (choc inélastique avec les molécules voisines) et de la variation de la section efficace d'absorption. Ainsi, les paramètres $\epsilon_{i, \text { las }}$ et $\phi_{i, \lambda}$ dépendent de la température dans l'Eq.(1) [CHA 16]. Expérimentalement, on observe que la dépendance à la température est exponentielle :

$$
I_{i, \lambda}(T)=K(\lambda) C_{i} V_{c} I_{l a s} e^{s_{i}(\lambda) T}
$$

où $K(\lambda)$ est un coefficient qui ne dépend que de la transmission du signal au détecteur. L'Eq.(2) est difficilement applicable à cause de biais de mesure liés aux variations de $K, V_{c}$ et $I_{\text {las }}$. Le passage d'une vague modifie l'épaisseur du film donc le volume $V_{c}$ de liquide éclairé, la réfraction de la lumière à la surface des vagues affecte la transmission du signal $(K)$ et le champ d'excitation laser $\left(I_{\text {las }}\right)$. Il est nécessaire d'utiliser une méthode ratiométrique comparant les intensités du signal de fluorescence sur deux bandes spectrales différentes. La méthode utilisée fait appels à deux colorants : les émissions de fluorescence mesurées sur chaque bande spectrale de détection proviennent d'un colorant différent [SAK 99]. Les colorants choisis, Kiton Red (KR) et Rhodamine 560 (Rh560), ont des concentrations respectives $C_{K R}=5 \cdot 10^{-8} \mathrm{~mol} / \mathrm{L}$ et $C_{R h 560}=5 \cdot 10^{-7} \mathrm{~mol} / \mathrm{L}$. La figure 3 montre que le signal de $\mathrm{KR}$ diminue lorsque la température augmente, alors que celui de Rh560 augmente lorsque la température augmente. Les deux bandes spectrales choisies sont [496-517] $\mathrm{nm}$ et [610-630] $\mathrm{nm}$ afin de minimiser les conflits spectraux entre les spectres d'absorption et d'émission des deux colorants [CHA 16]. En principe, le rapport des intensités de deux bandes de détection ne dépend que de la température seul 
(les phénomènes d'absorption et de réabsorption sont ici négligeables vu la faible épaisseur du film). Le rapport des intensités de fluorescence est donné par :

$$
R(T)=\frac{I_{1, \lambda_{1}}}{I_{2, \lambda_{2}}}=\frac{K\left(\lambda_{1}\right) \cdot C_{1}}{K\left(\lambda_{2}\right) \cdot C_{2}} e^{\left(s_{1}\left(\lambda_{1}\right)-s_{2}\left(\lambda_{2}\right)\right) T}=R\left(T_{r e f}\right) e^{s\left(T-T_{r e f}\right)},
$$

où le coefficient $s=s_{1}\left(\lambda_{1}\right)-s_{2}\left(\lambda_{2}\right)$ est la sensibilité en température du rapport de fluorescence. En pratique, la température est déterminée à partir de la variation du rapport $R$ par rapport à une référence prise préalablement à une température $T_{r e f}$ connue. Afin de mesurer l'intensité de fluorescence émise sur chaque bande spectrale, une sonde optique représentée en figure 2 a été développée. Le signal de fluorescence est induit par un laser continu $\mathrm{Nd}$ :YAG de longueur d'onde $532 \mathrm{~nm}$. Le faisceau laser est transmis par une fibre optique jusqu'à la sonde. Le faisceau laser est ensuite focalisé par une lentille convergente de courte focale afin d'illuminer le film sur toute son épaisseur $\delta$ par un faisceau cylindrique de diamètre environ $2 \mathrm{~mm}$. Le signal de fluorescence est ensuite collecté par la même lentille que la transmission du laser. Il est ensuite détecté par deux photomultiplicateurs, chacun équipé d'un filtre interférentiel correspondant à la bande spectrale sélectionnée. Une calibration effectuée à l'aide de cet arrangement optique donne une sensibilité à la température $s$ égale à $2,9 \% /{ }^{\circ} \mathrm{C}$. Le signal de fluorescence peut être également utilisé pour déterminer l'épaisseur $\delta$ du film en considérant directement les intensités de fluorescence des deux bandes. Lorsque le signal est de niveau équivalent sur les deux bandes de détection, la somme des intensités de ces deux bandes $\sum$ ne dépend pratiquement plus de la température, car les deux colorants ont des sensibilités très proches mais de signes opposés. Ainsi, on a :

$$
\sum=I_{1, \lambda}+I_{2, \lambda}=\sum_{r e f} \frac{\delta}{\delta_{r e f}}
$$

avec $\delta_{\text {ref }}$ une épaisseur de référence où la somme des signaux est connue. La dépendance à la température des deux bandes spectrales étant opposée, $\sum$ est peu affectée par la température (moins de $0,5 \% /{ }^{\circ} \mathrm{C}$ ). La sensibilité à la température de $\sum$ est négligeable, tandis que l'épaisseur du film a un très grand effet. $\sum$ est ainsi un paramètre de choix pour mesurer l'épaisseur du film. De même que pour la mesure de température, la prise d'une référence à une épaisseur $\delta_{r e f}$ connue permet de déterminer l'épaisseur instantanée du film. Le signal de chaque photomultiplicateur est numérisé par une carte d'acquisition à une fréquence fixée de $10 \mathrm{kHz}$.

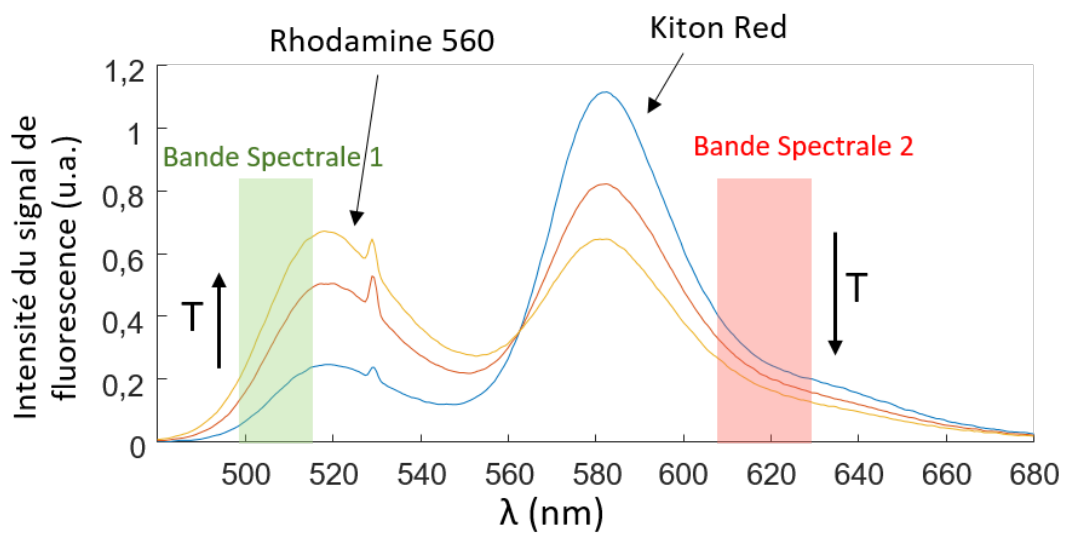

Figure 3. Spectre d'émission de fluorescence pour de l'eau ensemencée par Kiton Red et Rhodamine 560

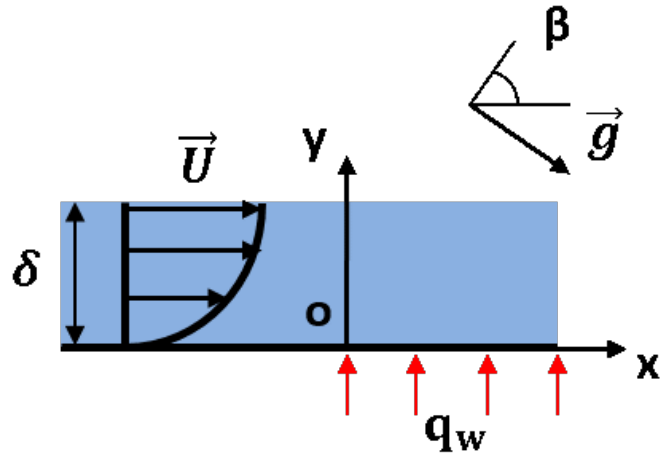

Figure 4. Schéma du film plat de la théorie de Nusselt 


\section{Résultats}

\subsection{Validation de la théorie du film plat de Nusselt}

Afin de valider le montage expérimental et les techniques de mesure, des tests de mesure ont été entrepris sur un film non perturbé. Sous certaines conditions de débit et d'inclinaison, un film non perturbé reste plat. Dans cette configuration, les résultats expérimentaux peuvent être comparés avec la théorie du film plat de Nusselt [NUS 16]. Cette configuration est présenté schématiquement en figure 4. Le film visqueux d'épaisseur $\delta$ s'écoule librement sur le plan incliné d'un angle $\beta$ par rapport à l'horizontal. En supposant un écoulement établi, les équations de Navier-Stokes donnent le champ de vitesse suivant dans le film :

$$
\vec{U}(y)=\frac{g \sin (\beta) \delta^{2}}{2 \nu}\left(\frac{2 y}{\delta}-\frac{y^{2}}{\delta^{2}}\right) \vec{x}
$$

avec $g$ l'accélération de la pesanteur et $\nu$ la viscosité cinématique du liquide. A partir de ce champ de vitesse, l'équation de la chaleur peut être résolue afin d'obtenir le champ de température [DAV 73]. On définit la température adimensionnée $\theta(x, y)=\frac{T(x, y)-T_{x=0}}{q_{w} \frac{\delta(x)}{k}}$, avec $q_{w}$ la densité de flux de chaleur à la paroi et $k$ la conductivité thermique du liquide. On introduit les longueurs adimensionnées $x^{*}=\frac{x}{\delta P e}$ et $y^{*}=\frac{y}{\delta}$ où $P e$ est le nombre de Peclet défini comme $P e=R e \cdot P r$, produit des nombres de Reynolds et de Prandtl. Ces nombres sont définis comme $R e=\frac{\Gamma}{\nu}, \operatorname{Pr}=\frac{\nu}{a}$ avec $\Gamma$ le débit volumique par unité de largeur et $a$ la diffusivité thermique du fluide. L'équation de la chaleur adimensionnée peut s'écrire :

$$
\left(2 y^{*}-y^{* 2}\right) \frac{\partial \theta}{\partial x^{*}}=\frac{\partial \theta}{\partial y^{*}}
$$

Les conditions limites correspondant à la configuration expérimentale sont :

$$
\left.\frac{\partial \theta}{\partial y^{*}}\right|_{y^{*}=0}=-\left.1 \quad \frac{\partial \theta}{\partial y^{*}}\right|_{y^{*}=1}=\left.0 \quad \theta\right|_{x^{*}=0}=0
$$

Ce problème peut se résoudre numériquement à l'aide d'un solveur d'équations aux dérivées partielles. Les mesures peuvent alors être comparées à ce modèle.

De manière générale, la température moyenne dans l'épaisseur du film peut être définie comme la température débitante :

$$
\overline{\theta_{f l}}=\frac{\int_{0}^{1} U \theta d y^{*}}{\int_{0}^{1} U d y^{*}}
$$

Cette température peut être déterminée expérimentalement à partir de la connaissance du flux de chaleur $q_{w}$ et du débit de liquide $\dot{Q}$.

En revanche, la température mesurée par la technique LIF à deux couleurs correspond à une moyenne volumique puisque le film liquide est éclairé dans toute son épaisseur :

$$
\overline{\theta_{v o l}}=\int_{0}^{1} \theta d y^{*}
$$

La figure 5 représente la comparaison entre les températures adimensionnées obtenues par calcul et expérience pour une densité du flux de chaleur $q_{w}=1,25 \mathrm{~W} / \mathrm{cm}^{2}$, un angle d'inclinaison $\beta=10^{\circ}$ 
et un débit volumique $\dot{Q}=4 \mathrm{1} / \mathrm{min}$ correspondant à un nombre de Reynolds $R e=200$. Sur cette figure, les températures débitantes $\theta_{f l}$ calculées par le modèle du film plat (ligne pleine) et à partir des mesures de flux de chaleur (points circulaires) sont représentées en rouge. En bleu, sont représentées les températures volumiques $\theta_{v o l}$ calculées par le modèle de film plat (ligne pleine) et par la technique $2 \mathrm{c}-\mathrm{LIF}$ sur le montage expérimental (points circulaires). Les résultats montrent un bon accord entre le modèle et l'expérience, confirmant que la sonde optique donne une mesure fiable de la température moyenne volumique.

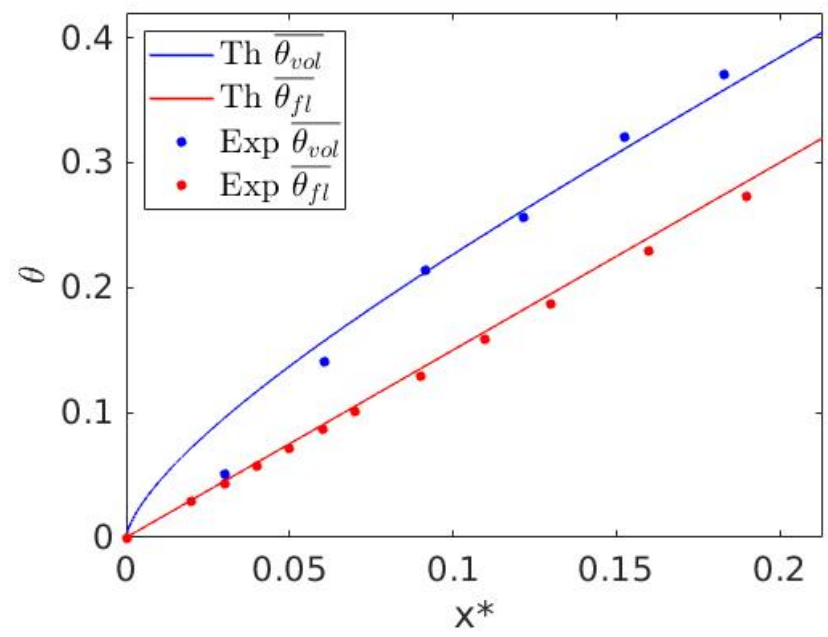

Figure 5. Comparaison de l'évolution spatiale des températures adimensionnées entre théorie (lignes) et expériences (points) pour $R e=200$, $\beta=10^{\circ}$ et $q_{w}=1,25 \mathrm{~W} / \mathrm{cm}^{2}$

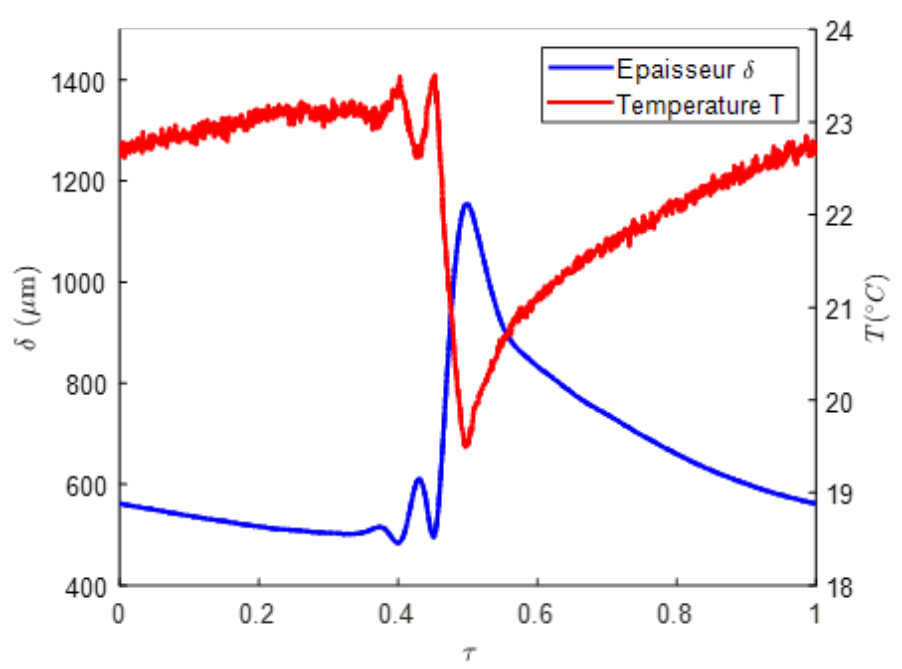

Figure 6. Mesures d'épaisseur et température LIF instantanées d'un film perturbé à une fréquence $f=5 \mathrm{~Hz}$ à $x=270 \mathrm{~mm}$ pour $R e=200, \beta=10^{\circ}$ et $q_{w}=1,25 \mathrm{~W} / \mathrm{cm}^{2}$

\subsection{Caractérisation de l'intensification des transferts de chaleur}

Afin de quantifier le transfert de chaleur et sa potentielle intensification due à la présence de vagues, des films perturbés de manière harmonique ont été étudiés. Grâce à la LIF, l'épaisseur $\delta$ ainsi que la température moyenne volumique $\overline{\theta_{\text {vol }}}$ sont mesurées simultanément avec une résolution temporelle de $10 \mathrm{kHz}$. La périodicité du forçage des vagues permet d'effectuer des moyennes de phase vague à vague, améliorant la précision de la mesure. En figure 6 sont représentées la température et l'épaisseur obtenues par moyenne de phase en fonction de $\tau=t f$, le temps adimensionné par la période des vagues $1 / f$. Ces mesures sont réalisées à une distance $x=270 \mathrm{~mm}$ du début de la zone chauffée du film. Les conditions expérimentales sont les mêmes que présentées en figure 5 avec l'ajout d'une perturbation de fréquence $f=5 \mathrm{~Hz}$. Dans ce tracé, une forte corrélation est observée entre l'épaisseur et la température du film. Plus le film liquide est mince, plus la température moyenne dans l'épaisseur du film est grande. Ce constat suggère une certaine stratification de la température dans l'épaisseur du film. Une intensification des transferts implique au contraire un mélange qui uniformise la température dans l'épaisseur du film.

Afin de caractériser l'intensification du transfert et mettre en évidence l'effet des vagues sur le champ de température, le nombre de Nusselt est défini par :

$$
N u=\frac{q_{w} \delta}{\left(T_{w}-T_{v o l}\right) k}
$$




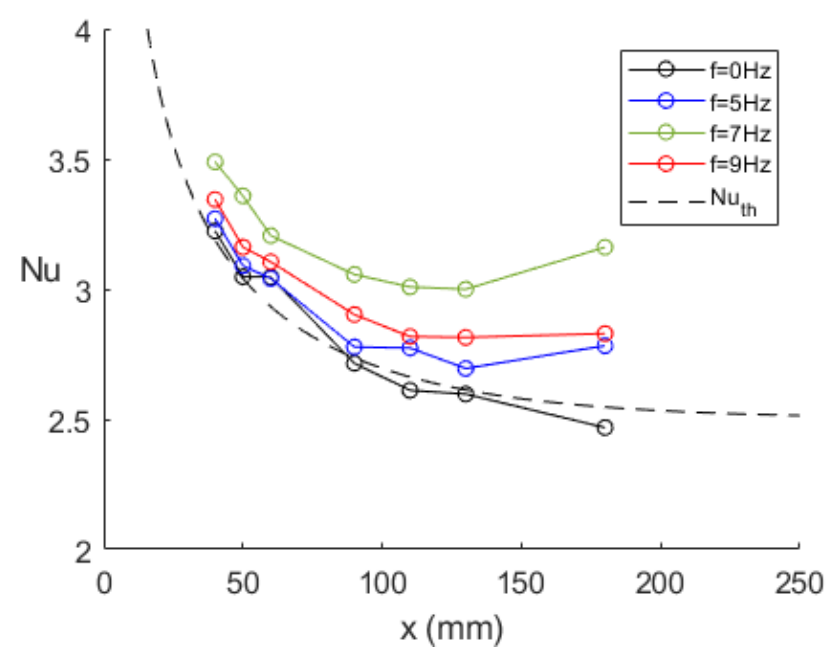

Figure 7. Évolution du nombre de Nusselt le long de l'écoulement en fonction de la fréquence des vagues pour $R e=200$ et $q_{w}=1,25 \mathrm{~W} / \mathrm{cm}^{2}$

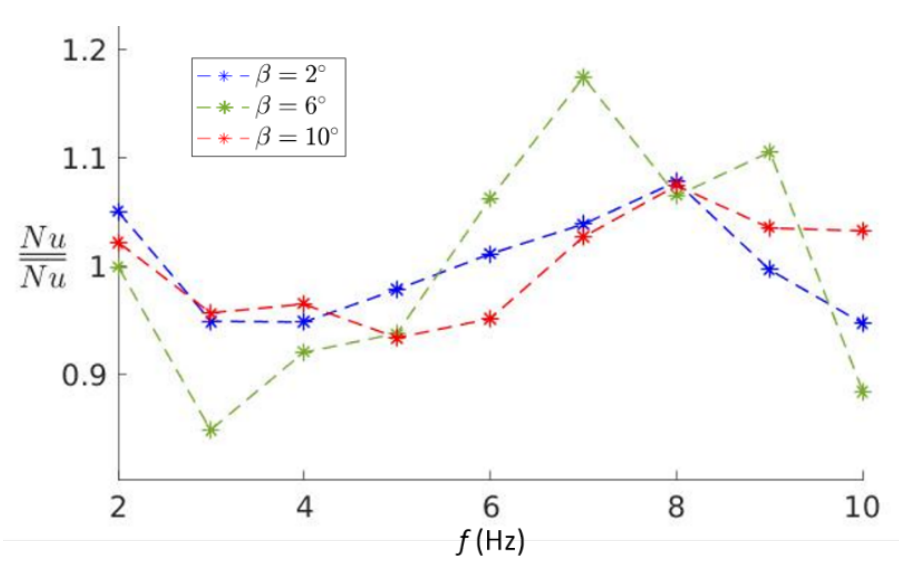

Figure 8. Effets de la fréquence des vagues sur les transferts de chaleur entre le film et le solide pour $x=270 \mathrm{~mm}, R e=200$ et $q_{w}=1,25 \mathrm{~W} / \mathrm{cm}^{2}$

avec $T_{w}$ la température de paroi locale et $T_{v o l}$ la température volumique du film moyennée au cours d'une période. Ce nombre compare le rapport entre les transferts convectif et conductif au sein du film. La figure 7 représente l'évolution spatiale du nombre de Nusselt $N u$ pour différentes fréquences de vagues, comparée à la solution théorique issue du modèle de film plat de Nusselt. La principale incertitude sur $N u$ provient de la mesure de température de paroi $T_{w}$, par thermocouple avec une incertitude de $\pm 0.5^{\circ}$, ce qui conduit à une incertitude sur $N u$ de \pm 0.1 . Cette représentation montre que la présence des vagues tend bien à intensifier les transferts de chaleur par convection à travers le film et que leur fréquence a une influence sur l'intensification, qui est maximale pour la fréquence d'excitation $f=7 \mathrm{~Hz}$. Suggérant que la présence de vague induit un mélange du fluide dans le film uniformisant le champ de température, la présence d'une poche de recirculation dans la crête de la vague pourrai expliquer le phénomène. En effet, le développement des vagues au cours de l'écoulement leur permettrait de soutirer du liquide proche de la paroi afin d'alimenter la crête de vague. Ce faisant le gradient de température proche de la paroi chaude serai augmenté, facilitant les transfert de chaleur.

Afin de comparer les effets de la fréquence des vagues pour différents angles d'inclinaison de la paroi, le nombre de Nusselt $N u$ est normalisé par $\overline{N u}$, la valeur moyenne des $N u$ de la série de mesures à inclinaison fixée considérée. Cette normalisation des différentes séries de mesures à inclinaison fixée permet de comparer l'évolution fréquentielle du Nombre de Nusselt $N u$ pour différentes inclinaisons. Les résultats sont présentés en figure 8 , les conditions expérimentales sont les mêmes que précédemment : $q_{w}=1,25 \mathrm{~W} / \mathrm{cm}^{2}, x=270 \mathrm{~mm}$ et $R e=200$, la fréquence d'excitation $f$ est contrôlée de 0 à $10 \mathrm{~Hz}$ et l'angle d'inclinaison $\beta$ varie de 2 à $10^{\circ}$. Les mesures montrent que le transfert de chaleur a tendance à être plus important lorsque la fréquence des vagues est autour de 6 ou $7 \mathrm{~Hz}$ et diminue lorsque la fréquence est faible, autour de 3 ou $4 \mathrm{~Hz}$. En particulier, l'effet de la fréquence d'excitation est le plus marqué à $l^{\prime}$ inclinaison de $6^{\circ}$. Cette figure montre que la fréquence des vagues a un effet notoire sur les transferts de chaleur comme mentionné dans [FRI 72]. La fréquence d'excitation du film influe sur la périodicité des vagues, tandis que l'inclinaison de la paroi influe sur l'épaisseur moyenne du film et l'amplitude des vagues relative à cette épaisseur de film. Ainsi, ces deux paramètres ont une influence sur la taille et la forme des crêtes de vague où peuvent se développer des poches de recirculation et modifient le mélange 
advectif pouvant se mettre en place à l'intérieur du film perturbé. Si l'effet d'intensification provient des recirculations transportées par les vagues qui uniformisent le champ de température, cela signifierait qu'il existe des fréquences plus propices à leur apparition et à leur développement et que certaines structures permettent un mélange plus efficace du fluide à l'intérieur de la vague. Dans le futur, le lien entre ces résultats et la description temporelle des vagues montrée en figure 6 permettra d'expliquer cette tendance.

\section{Conclusion}

Dans cette étude, les transferts de chaleur dans un film liquide ont été étudiés. Les résultats concernent en particulier le cas d'un film perturbé harmoniquement s'écoulant sur un plan incliné chauffé. L'originalité de ce travail vient de l'utilisation de la fluorescence induite par laser apportant une mesure couplée de la température du film et de son épaisseur, qui de plus est résolue dans le temps. La technique de mesure a été validée par une comparaison avec le cas de film plat théorique de Nusselt. Les premiers résultats obtenus montrent une claire corrélation entre l'épaisseur du film et le nombre de Nusselt local. Cette information résolue dans le temps rendra possible la liaison entre la topologie de la vague et ses effets sur les transferts de chaleur afin de tendre vers une meilleure méthode de contrôle de l'intensification des transferts dans un film mince en étendant l'étude paramétrique au nombre de Reynolds par exemple. De plus, l'étude de la distribution du champ de température dans l'épaisseur du film par une technique de fluorescence induite par laser étendue à de l'imagerie dans le plan permettra de comprendre les mécanismes fins des transferts de chaleur entre le film et la paroi.

\section{Remerciements}

Ce travail a été financé par l'Agence Nationale de la Recherche (ANR) dans le cadre du projet FRAISE (Film Ruisselants à Instabilités de Surface : Exploration, AAP-2016).

\section{Bibliographie}

[ALS 02] F. Al-Sibai, A. Leefken and U. Renz. Local and instantaneous distribution of heat transfer rates through wavy films, International Journal of Thermal Sciences, 41(7) :658 - 663, 2002.

[CHA 16] W. Chaze, O. Caballina, G. Castanet, and F. Lemoine. The saturation of the fluorescence and its consequences for laser-induced fluorescence thermometry in liquid flows, Experiments in Fluids, 57(4), 2016.

[CHA 17] W. Chaze, O. Caballina, G. Castanet, and F. Lemoine. Spatially and temporally resolved measurements of the temperature inside droplets impinging on a hot solid surface, Experiments in Fluids, 58(98), 2017.

[DAV 73] E.J. Davis. Exact solutions for a class of heat and mass transfer problems, The Canadian Journal of Chemical Engineering, 51(5) :562-572, 1973.

[FRI 72] D.P. Frisk and E.J. Davis. The enhancement of heat transfer by waves in stratifiedgas-liquid flow, International Journal of Heat and Mass Transfer. 15(8):1537 - 1552,1972.

[LEM 13] F. Lemoine and G. Castanet. Temperature and chemical composition of droplets by optical measurement techniques : A state-of-the-art review, Experiments in Fluids, 54(1572), 2013.

[MAR 16] C.N. Markides, R. Mathie and A. Charogiannis. An experimental study of spatiotemporally resolved heat transfer in thin liquid-film flows falling over an inclined heated foil, International Journal of Heat and Mass Transfer, $93: 872$ - 888, 2016.

[MAT 13] R. Mathie, H. Nakamura and C.N. Markides. Heat transfer augmentation in unsteady conjugate thermal systems - Part II : Applications, International Journal of Heat and Mass Transfer, 56(1) :819 - 833, 2013. 
[NUS 16] W. Nusselt. Die Oberflächenkondensation des Wasserdampfe, VDI, 1916.

[POR 71] S. Portalski and A.J. Clegg. Interfacial area increase in rippled film flow on wetted wallcolumns, Chemical Engineering Science., 26(6) :773 - 784, 1971.

[RUY 14] C. Ruyer-Quil, N. Kofman, D. Chasseur and S. Mergui. Dynamics of falling liquid films, The European Physical Journal. 37(4) :30, 2014.

[SAK 99] J. Sakakibara and R. J. Adrian. Whole field measurement of temperature in water using two-color laser induced fluorescence, Experiments in Fluids, 26(1):7-15, 1999.

[SCH 06] A. Schagen, M. Modigell, G. Dietze and R. Kneer. Simultaneous measurement of local film thickness and temperature distribution in wavy liquid films using a luminescence technique, International Journal of Heat and Mass Transfer, 49(25):5049 - 5061, 2006.

[SCH 07] A. Schagen and M. Modigell. Local film thickness and temperature distribution measurement in wavy liquid films with a laser-induced luminescence technique, Experiments in Fluids, 43(2) :209 - 221, 2007.

[STI 19] M. Stiti, A. Labergue, F. Lemoine, S. Leclerc, D. Stemmelen.Temperature measurement and state determination of supercooled droplets using laser-induced fluorescence, Experiments in Fluids, 60(69), 2019. 